\title{
Experimental Study of Totally Optimal Decision Trees
}

\author{
Abdulla Aldilaijan \\ Brown University \\ Department of Computer Science \\ Providence, Rhode Island 02912 USA \\ abdulla_aldilaijan@brown.edu
}

Mohammad Azad, Mikhail Moshkov

Computer, Electrical and Mathematical Sciences \& Engineering Division

King Abdullah University of Science and Technology

Thuwal 23955-6900, Saudi Arabia

\{mohammad.azad, mikhail.moshkov\}@kaust.edu.sa

\begin{abstract}
In this paper, we present results of experimental studies related to the existence of totally optimal decision trees (which are optimal relative to two or more cost functions simultaneously) for nine decision tables from the UCI Machine Learning Repository. Such trees can be useful when we consider decision trees as algorithms for problem solving or as a way for knowledge representation. For cost functions, we use depth, average depth, and number of nodes. We study not only exact but also approximate decision trees based on five uncertainty measures: entropy, Gini index, misclassification error, relative misclassification error, and number of unordered pairs of rows with different decisions. To investigate the existence of totally optimal trees, we use an extension of dynamic programming that allows us to make multi-stage optimization of decision trees relative to a sequence of cost functions. Experimental results show that totally optimal decision trees exist in many cases. The behavior of graphs that describe how the number of decision tables with totally optimal decision trees depends on their accuracy is mainly
\end{abstract}

Address for correspondence: Computer, Electrical and Mathematical Sciences \& Engineering Division King Abdullah University of Science and Technology 
irregular. However, one can observe some trends, in particular, an upward trend when accuracy is decreasing.

Keywords: decision tree, uncertainty measure, cost function, totally optimal decision tree, multistage optimization

\section{Introduction}

In this paper, we experimentally investigate the existence of the totally optimal decision trees that are optimal relative to two or more cost functions simultaneously, for example, have in the same time minimum depth and minimum number of nodes. Such decision trees can be useful if they are considered as algorithms for problem solving (see, for example, [1]) or as a way for knowledge representation (see, for example, [2, 3]). When decision trees are considered as algorithms for problem solving, we would like to simultaneously minimize its time complexity (depth or/and average depth) and its space complexity (number of nodes). When decision trees are considered as a way for knowledge representation, we would like to simultaneously minimize the number of nodes (to make the decision tree more understandable) and the depth (to make understandable conjunctions of conditions corresponding to the paths from the root to terminal nodes). We show that such decision trees exist in many cases.

We have a powerful tool for the study of totally optimal decision trees based on extensions of dynamic programming: the multi-stage optimization of decision trees relative to a sequence of criteria [4]. For experimenting, we use Dagger system created in KAUST to implement various extensions of dynamic programming [5, 6].

For cost functions, we consider the depth $h$, average depth $h_{\text {avg }}$, and number of nodes $L$ which describe the time complexity in the worst case, time complexity in the average case, and space complexity of decision trees, respectively. We study totally optimal decision trees relative to each pair of the considered cost functions and totally optimal decision trees relative to the three cost functions.

We study not only exact but also approximate decision trees defined based on five uncertainty measures: entropy ent, Gini index gini, misclassification error me, relative misclassification error rme, and number of unordered pairs of rows with different decisions $R$. Let $U$ be an uncertainty measure and $\alpha$ be a real number such that $0 \leq \alpha \leq 1$. We consider $(U, \alpha)$-decision trees for a decision table $T$ in which, for each terminal node of the tree, the uncertainty of the subtable of $T$ corresponding to this node is at most $\alpha U(T)$ where $U(T)$ is the uncertainty of the decision table $T$ and, for each nonterminal node, the uncertainty of the subtable of $T$ corresponding to this node is greater than $\alpha U(T)$. For a fixed uncertainty measure $U$, the parameter $\alpha$ is related to the accuracy of decision trees. If $\alpha=0$, then we deal with exact decision trees. If $\alpha>0$, then we have in general case approximate decision trees. If $\alpha$ is increasing then in general case the accuracy of decision trees is decreasing.

One of the main goals of this paper is to experimentally study how the existence of totally optimal $(U, \alpha)$-decision trees depends on $\alpha$. We work with nine decision tables from the UCI Machine Learning Repository [7]. Experimental results show that totally optimal $(U, \alpha)$-decision trees exist in many cases. The behavior of graphs that describe how the number of decision tables with totally optimal 
$(U, \alpha)$-decision trees depends on $\alpha$ is mainly irregular. However, we notice an upward trend when $\alpha$ is increasing.

One of the main areas of applications of the obtained results (existence of the totally optimal decision trees in many cases) is the rough set theory $[8,9]$ in which decision trees and rules are widely used. Decision trees that are totally optimal relative to the number of nodes and depth or average depth can be used for knowledge representation. Decision trees that are totally optimal relative to the number of terminal nodes and depth can be used for the design of the decision rule systems. The number of decision rules derived from a decision tree (each rule corresponds to a path from the root to a terminal node of the tree) is equal to the number of terminal nodes in the decision tree, and the maximum length of the derived rules is equal to the depth of the tree.

Note that totally optimal decision trees computing Boolean functions were considered in [10] including exact and approximate decision trees defined based on relative misclassification error $r m e$ uncertainty measure. The following two statements were proved in this paper:

- As $n \rightarrow \infty$, almost all Boolean functions with $n$ variables have totally optimal exact decision trees relative to the depth and number of nodes.

- As $n \rightarrow \infty$, almost all Boolean functions with $n$ variables have totally optimal exact decision trees relative to the depth and average depth.

Also note that the totally optimal exact decision trees for decision tables with many-valued decisions relative to different combinations of cost functions were studied in [11].

This paper is an extended version of the conference paper [12]. We explained in detail tools for the study of totally optimal decision trees and added more experimental results.

This paper consists of five sections. In Sect. 2, we consider main notions. In Sect. 3, we discuss tools for the study of totally optimal decision trees. Section 4 is devoted to the consideration of experimental results. Section 4 contains short conclusions.

\section{Main Notions}

In this section, we discuss the notions of decision table, uncertainty measure, decision tree, cost function, and totally optimal decision tree.

\subsection{Decision Tables}

A decision table is a rectangular table $T$ with $n \geq 1$ columns filled with numbers from the set $\omega=$ $\{0,1,2, \ldots\}$ of nonnegative integers. Columns of the table are labeled with conditional attributes $f_{1}, \ldots, f_{n}$. Rows of the table are pairwise different, and each row is labeled with a number from $\omega$ which is interpreted as a decision (a value of the decision attribute $d$ ). Rows of the table are interpreted as tuples of values of conditional attributes. We denote by $\mathcal{T}$ the set of all decision tables. An example of a decision table can be found in Fig. 1.

A decision table is called empty if it has no rows. The table $T$ is called degenerate if it is empty or all rows of $T$ are labeled with the same decision. Let $D(T)$ be the set of decisions attached to rows of 


$T_{0}=$\begin{tabular}{|rrr|r|}
\hline$f_{1}$ & $f_{2}$ & $f_{3}$ & \\
\hline 0 & 1 & 1 & 1 \\
0 & 0 & 0 & 2 \\
1 & 0 & 1 & 3 \\
\hline
\end{tabular}

Figure 1. Decision table $T_{0}$ with three conditional attributes and three rows

$T$. We denote by $N(T)$ the number of rows in the table $T$ and, for any $t \in \omega$, we denote by $N_{t}(T)$ the number of rows of $T$ labeled with the decision $t$. By $\operatorname{mcd}(T)$ we denote the most common decision for $T$ which is the minimum decision $t_{0}$ from $D(T)$ such that $N_{t_{0}}(T)=\max \left\{N_{t}(T): t \in D(T)\right\}$. If $T$ is empty then $\operatorname{mcd}(T)=0$.

For any conditional attribute $f_{i} \in\left\{f_{1}, \ldots, f_{n}\right\}$, we denote by $E\left(T, f_{i}\right)$ the set of values of the attribute $f_{i}$ in the table $T$. We denote by $E(T)$ the set of conditional attributes for which $\left|E\left(T, f_{i}\right)\right| \geq$ 2 .

Let $T$ be a nonempty decision table. A subtable of $T$ is a table obtained from $T$ by removal of some rows. Let $f_{i_{1}}, \ldots, f_{i_{m}} \in\left\{f_{1}, \ldots, f_{n}\right\}$ and $a_{1}, \ldots, a_{m} \in \omega$. We denote by $T\left(f_{i_{1}}, a_{1}\right) \ldots\left(f_{i_{m}}, a_{m}\right)$ the subtable of the table $T$ containing the rows from $T$ which at the intersection with the columns $f_{i_{1}}, \ldots, f_{i_{m}}$ have numbers $a_{1}, \ldots, a_{m}$, respectively. Such nonempty subtables, including the table $T$, are called separable subtables of $T$.

\subsection{Uncertainty Measures}

Let $\mathbb{R}$ be the set of real numbers and $\mathbb{R}_{+}$be the set of all nonnegative real numbers. An uncertainty measure is a function $U: \mathcal{T} \rightarrow \mathbb{R}$ such that $U(T) \geq 0$ for any $T \in \mathcal{T}$, and $U(T)=0$ if and only if $T$ is a degenerate table. The following functions (we assume that, for any empty table, the value of each of the considered functions is equal to 0 ) are uncertainty measures:

- Misclassification error $m e(T)=N(T)-N_{m c d(T)}(T)$.

- Relative misclassification error $r m e(T)=\left(N(T)-N_{m c d(T)}(T)\right) / N(T)$.

- Entropy $\operatorname{ent}(T)=-\sum_{t \in D(T)}\left(N_{t}(T) / N(T)\right) \log _{2}\left(N_{t}(T) / N(T)\right)$.

- Gini index $\operatorname{gini}(T)=1-\sum_{t \in D(T)}\left(N_{t}(T) / N(T)\right)^{2}$.

- Function $R$ where $R(T)$ is the number of unordered pairs of rows of $T$ labeled with different decisions (note that $R(T)=N(T)^{2} \operatorname{gini}(T) / 2$ ).

For the decision table $T_{0}$ depicted in Fig. $1, \operatorname{me}\left(T_{0}\right)=2, \operatorname{rme}\left(T_{0}\right)=2 / 3$, ent $\left(T_{0}\right)=\log _{2} 3$, $\operatorname{gini}\left(T_{0}\right)=2 / 3$, and $R\left(T_{0}\right)=3$. 


\subsection{Decision Trees}

Let $T$ be a decision table with $n$ conditional attributes $f_{1}, \ldots, f_{n}$.

A decision tree over $T$ is a finite directed tree with root in which nonterminal nodes are labeled with attributes from the set $\left\{f_{1}, \ldots, f_{n}\right\}$, terminal nodes are labeled with numbers from $\omega$, and, for each nonterminal node, edges starting from this node are labeled with pairwise different numbers from $\omega$.

Let $\Gamma$ be a decision tree over $T$ and $v$ be a node of $\Gamma$. We define now a subtable $T(v)=T_{\Gamma}(v)$ of the table $T$. If $v$ is the root of $\Gamma$ then $T(v)=T$. Let $v$ be not the root of $\Gamma$ and $v_{1}, e_{1}, \ldots, v_{m}, e_{m}, v_{m+1}=$ $v$ be the directed path from the root of $\Gamma$ to $v$ in which nodes $v_{1}, \ldots, v_{m}$ are labeled with attributes $f_{i_{1}}, \ldots, f_{i_{m}}$ and edges $e_{1}, \ldots, e_{m}$ are labeled with numbers $a_{1}, \ldots, a_{m}$, respectively. Then $T(v)=$ $T\left(f_{i_{1}}, a_{1}\right) \ldots\left(f_{i_{m}}, a_{m}\right)$.

Let $U$ be an uncertainty measure and $\alpha$ be a real number such that $0 \leq \alpha \leq 1$.

A decision tree $\Gamma$ over $T$ is called a $(U, \alpha)$-decision tree for $T$ if, for any node $v$ of $\Gamma$,

- If $U(T(v)) \leq \alpha U(T)$ then $v$ is a terminal node which is labeled with the number $m c d(T(v))$.

- If $U(T(v))>\alpha U(T)$ then $v$ is a nonterminal node labeled with an attribute $f_{i} \in E(T(v))$, and if $E\left(T(v), f_{i}\right)=\left\{a_{1}, \ldots, a_{t}\right\}$ then $t$ edges start from the node $v$ which are labeled with $a_{1}, \ldots, a_{t}$, respectively.

We denote by $D T_{U, \alpha}(T)$ the set of $(U, \alpha)$-decision tree for $T$. Let $\alpha, \beta$ be real numbers such that $0 \leq \alpha<\beta \leq 1$ and $\Gamma$ be a $(U, \alpha)$-decision tree for $T$. Then it is possible that $\Gamma$ is not a $(U, \beta)$-decision tree for $T$.

An example of $(m e, 0)$-decision tree $\Gamma_{0}$ for the decision table $T_{0}$ depicted in Fig. 1 can be found in Fig. 2.

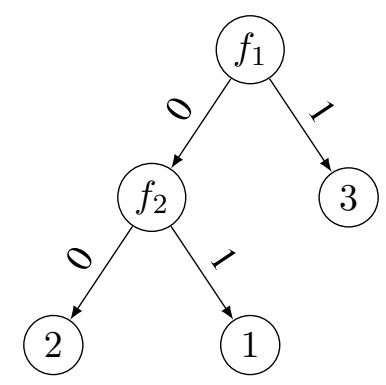

Figure 2. $(m e, 0)$-Decision tree $\Gamma_{0}$ for decision table $T_{0}$

\subsection{Cost Functions}

Let $n$ be a natural number. We consider a partial order $\leq$ on the set $\mathbb{R}^{n}:\left(x_{1}, \ldots, x_{n}\right) \leq\left(y_{1}, \ldots, y_{n}\right)$ if $x_{1} \leq y_{1}, \ldots, x_{n} \leq y_{n}$. A function $g: \mathbb{R}_{+}^{n} \rightarrow \mathbb{R}_{+}$is called increasing if $g(x) \leq g(y)$ for any $x, y \in \mathbb{R}_{+}^{n}$ such that $x \leq y$. A function $g: \mathbb{R}_{+}^{n} \rightarrow \mathbb{R}_{+}$is called strictly increasing if $g(x)<g(y)$ for 
any $x, y \in \mathbb{R}_{+}^{n}$ such that $x \leq y$ and $x \neq y$. If $g$ is strictly increasing then, evidently, $g$ is increasing. For example, $\max \left(x_{1}, x_{2}\right)$ is increasing and $x_{1}+x_{2}$ is strictly increasing.

Let $f$ be a function from $\mathbb{R}_{+}^{2}$ to $\mathbb{R}_{+}$. We can extend $f$ to a function with arbitrary number of variables in the following way: $f\left(x_{1}\right)=x_{1}$ and, if $n>2$ then $f\left(x_{1}, \ldots, x_{n}\right)=f\left(f\left(x_{1}, \ldots, x_{n-1}\right), x_{n}\right)$.

If $f$ be an increasing function from $\mathbb{R}_{+}^{2}$ to $\mathbb{R}_{+}$then, for any natural $n$, the function $f\left(x_{1}, \ldots, x_{n}\right)$ is increasing. If $f$ is a strictly increasing function from $\mathbb{R}_{+}^{2}$ to $\mathbb{R}_{+}$then, for any natural $n$, the function $f\left(x_{1}, \ldots, x_{n}\right)$ is strictly increasing.

For $b \in \omega$, we denote by tree $(b)$ the decision tree that contains only one (terminal) node labeled with $b$. Let $f_{i} \in\left\{f_{1}, \ldots, f_{n}\right\}, a_{1}, \ldots, a_{t}$ be pairwise different numbers from $\omega$, and $\Gamma_{1}, \ldots, \Gamma_{t}$ be decision trees over $T$. We denote by $\operatorname{tree}\left(f_{i}, a_{1}, \ldots, a_{t}, \Gamma_{1}, \ldots, \Gamma_{t}\right)$ the following decision tree over $T$ : the root of the tree is labeled with $f_{i}$, and $t$ edges start from the root which are labeled with $a_{1}, \ldots, a_{t}$ and enter the roots of decision trees $\Gamma_{1}, \ldots, \Gamma_{t}$, respectively.

A cost function for decision trees is a function $\psi(T, \Gamma)$ which is defined on pairs of decision table $T$ and a decision tree $\Gamma$ for $T$, and has values from $\mathbb{R}_{+}$. The function $\psi$ is given by three functions $\psi^{0}: \mathcal{T} \rightarrow \mathbb{R}_{+}, F: \mathbb{R}_{+}^{2} \rightarrow \mathbb{R}_{+}$and $w: \mathcal{T} \rightarrow \mathbb{R}_{+}$.

The value of $\psi(T, \Gamma)$ is defined by induction:

- If $\Gamma=\operatorname{tree}(\operatorname{mcd}(T))$ then $\psi(T, \Gamma)=\psi^{0}(T)$.

- If $\Gamma=\operatorname{tree}\left(f_{i}, a_{1}, \ldots, a_{t}, \Gamma_{1}, \ldots, \Gamma_{t}\right)$ then

$$
\psi(T, \Gamma)=F\left(\psi\left(T\left(f_{i}, a_{1}\right), \Gamma_{1}\right), \ldots, \psi\left(T\left(f_{i}, a_{t}\right), \Gamma_{t}\right)\right)+w(T) .
$$

The cost function $\psi$ is called increasing if $F$ is an increasing function. The cost function $\psi$ is called strictly increasing if $F$ is a strictly increasing function.

We now consider examples of cost functions for decision trees:

- Depth $h(T, \Gamma)=h(\Gamma)$ of a decision tree $\Gamma$ for a decision table $T$ is the maximum length of a path in $\Gamma$ from the root to a terminal node. For this cost function, $\psi^{0}(T)=0, F(x, y)=$ $\max (x, y)$, and $w(T)=1$. This is an increasing cost function.

- Total path length $\operatorname{tpl}(T, \Gamma)$ of a decision tree $\Gamma$ for a decision table $T$ is equal to $\sum_{r \in \operatorname{Row}(T)} l_{\Gamma}(r)$ where $\operatorname{Row}(T)$ is the set of rows of $T$, and $l_{\Gamma}(r)$ is the length of a path in $\Gamma$ from the root to a terminal node $v$ such that the row $r$ belongs to $T_{\Gamma}(v)$. For this cost function, $\psi^{0}(T)=0$, $F(x, y)=x+y$, and $w(T)=N(T)$. This is a strictly increasing cost function. Let $T$ be a nonempty decision table. The value $h_{\text {avg }}(T, \Gamma)=\operatorname{tpl}(T, \Gamma) / N(T)$ is the average depth of a decision tree $\Gamma$ for a decision table $T$.

- Number of nodes $L(T, \Gamma)=L(\Gamma)$ of a decision tree $\Gamma$ for a decision table $T$. For this cost function, $\psi^{0}(T)=1, F(x, y)=x+y$, and $w(T)=1$. This is a strictly increasing cost function.

For the decision table $T_{0}$ depicted in Fig. 1 and the decision tree $\Gamma_{0}$ for $T_{0}$ depicted in Fig. 2, $h\left(\Gamma_{0}\right)=2, \operatorname{tpl}\left(T_{0}, \Gamma_{0}\right)=5, h_{\text {avg }}\left(T_{0}, \Gamma_{0}\right)=5 / 3$, and $L\left(\Gamma_{0}\right)=5$. 


\subsection{Totally Optimal Decision Trees}

Let $U$ be an uncertainty measure, $\alpha$ be a real number such that $0 \leq \alpha \leq 1$, and $T$ be a decision table.

For a cost function $\psi$, we denote $\psi^{U, \alpha}(T)=\min \left\{\psi(T, \Gamma): \Gamma \in D T_{U, \alpha}(T)\right\}$, i.e., $\psi^{U, \alpha}(T)$ is the minimum cost of a $(U, \alpha)$-decision tree for $T$ relative to the cost function $\psi$. Let $\psi_{1}, \ldots, \psi_{m}$ be cost functions and $m \geq 2$.

A $(U, \alpha)$-decision tree $\Gamma$ for $T$ is called a totally optimal $(U, \alpha)$-decision tree for $T$ relative to the cost functions $\psi_{1}, \ldots, \psi_{m}$ if $\psi_{1}(T, \Gamma)=\psi_{1}^{U, \alpha}(T), \ldots, \psi_{m}(T, \Gamma)=\psi_{m}^{U, \alpha}(T)$, i.e., $\Gamma$ is optimal relative to $\psi_{1}, \ldots, \psi_{m}$ simultaneously.

One can show that the decision tree $\Gamma_{0}$ depicted in Fig. 2 is a totally optimal ( $\left.m e, 0\right)$-decision tree for the decision table $T_{0}$ depicted in Fig. 1 relative to $h, h_{\text {avg }}$, and $L$.

Let us consider another example of a totally optimal decision tree. The disjunction $x_{1} \vee x_{2} \vee$ $x_{3}$ can be represented as a decision table with three conditional attributes $x_{1}, x_{2}, x_{3}$ and eight rows corresponding to tuples from $\{0,1\}^{3}$ that are labeled with decisions - values of $x_{1} \vee x_{2} \vee x_{3}$ on these tuples. One can show that the decision tree depicted in Fig. 3 is a totally optimal $(m e, 0)$-decision tree for the considered decision table relative to $h, h_{\text {avg }}$, and $L$. This is a totally optimal decision tree relative to $h, h_{\text {avg }}$, and $L$ computing disjunction $x_{1} \vee x_{2} \vee x_{3}$.

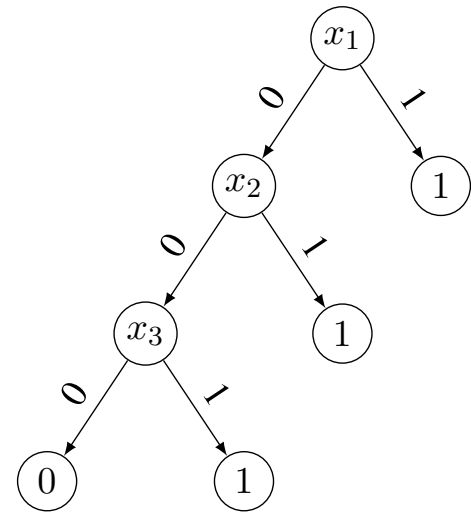

Figure 3. Totally optimal decision tree relative to $h, h_{\text {avg }}$, and $L$ computing disjunction $x_{1} \vee x_{2} \vee x_{3}$

\section{Tools for Study of Totally Optimal Decision Trees}

In this section, we consider an algorithm for construction of a directed acyclic graph (DAG) $\Delta_{U, \alpha}(T)$ which describes all $(U, \alpha)$-decision trees for a decision table $T$, and consider an algorithm for optimization of $(U, \alpha)$-decision trees for $T$ relative to a cost function. We discuss also a way to prove experimentally the existence of totally optimal $(U, \alpha)$-decision trees for $T$ based on results of multistage optimization of decision trees. 


\subsection{Directed Acyclic Graph $\Delta_{U, \alpha}(T)$}

Let $T$ be a nonempty decision table with $n$ conditional attributes $f_{1}, \ldots, f_{n}, U$ be an uncertainty measure, and $\alpha$ be a real number such that $0 \leq \alpha \leq 1$. We now consider an algorithm $\mathcal{A}_{1}$ described in [13] for the construction of a directed acyclic graph $\Delta_{U, \alpha}(T)$ which will be used for the description and optimization of $(U, \alpha)$-decision trees for $T$. Nodes of this graph are some separable subtables of the table $T$. During each iteration, we process one node. We start with the graph that consists of one node $T$ which is not processed and finish when all nodes of the graph are processed.

Algorithm $\mathcal{A}_{1}$ (construction of DAG $\Delta_{U, \alpha}(T)$ ).

Input: A nonempty decision table $T$ with $n$ conditional attributes $f_{1}, \ldots, f_{n}$, an uncertainty measure $U$, and real number $\alpha$ such that $0 \leq \alpha \leq 1$.

Output: Directed acyclic graph $\Delta_{U, \alpha}(T)$.

1. Construct the graph that consists of one node $T$ which is not marked as processed.

2. If all nodes of the graph are processed then the work of the algorithm is finished. Return the resulting graph as $\Delta_{U, \alpha}(T)$. Otherwise, choose a node (table) $\Theta$ that has not been processed yet.

3. If $U(\Theta) \leq \alpha U(T)$ mark the node $\Theta$ as processed and proceed to step 2 .

4. If $U(\Theta)>\alpha U(T)$ then, for each $f_{i} \in E(\Theta)$, draw a bundle of edges from the node $\Theta$ (this bundle of edges will be called $f_{i}$-bundle). Let $E\left(\Theta, f_{i}\right)=\left\{a_{1}, \ldots, a_{k}\right\}$. Then draw $k$ edges from $\Theta$ and label these edges with the pairs $\left(f_{i}, a_{1}\right), \ldots,\left(f_{i}, a_{k}\right)$. These edges enter nodes $\Theta\left(f_{i}, a_{1}\right), \ldots, \Theta\left(f_{i}, a_{k}\right)$, respectively. If some of the nodes $\Theta\left(f_{i}, a_{1}\right), \ldots, \Theta\left(f_{i}, a_{k}\right)$ are not present in the graph then add these nodes to the graph. Mark the node $\Theta$ as processed and return to step 2 .

An example of the directed acyclic graph $\Delta_{m e, 0}\left(T_{1}\right)$ for a simple decision table $T_{1}$ with two conditional attributes and four rows can be found in Fig. 4 (the decision table $T_{1}$ is at the top of this figure).

A node of directed graph is called terminal if there are no edges starting in this node. A bundlepreserving subgraph of the graph $\Delta_{U, \alpha}(T)$ is a graph $G$ obtained from $\Delta_{U, \alpha}(T)$ by removal of some bundles of edges such that each nonterminal node of $\Delta_{U, \alpha}(T)$ keeps at least one bundle of edges starting in this node. By definition, $\Delta_{U, \alpha}(T)$ is a bundle-preserving subgraph of $\Delta_{U, \alpha}(T)$. A node $\Theta$ of the graph $G$ is terminal if and only if $U(\Theta) \leq \alpha U(T)$.

Let $G$ be a bundle-preserving subgraph of the graph $\Delta_{U, \alpha}(T)$. For each nonterminal node $\Theta$ of the graph $G$, we denote by $E_{G}(\Theta)$ the set of attributes $f_{i}$ from $E(\Theta)$ such that $f_{i}$-bundle of edges starts from $\Theta$ in $G$. For each terminal node $\Theta, E_{G}(\Theta)=\emptyset$. For each node $\Theta$ of the graph $G$, we define the set $\operatorname{Tree}(G, \Theta)$ of decision trees in the following way. If $\Theta$ is a terminal node of $G$ (in this case $U(\Theta) \leq \alpha U(T)$ ), then $\operatorname{Tree}(G, \Theta)=\{\operatorname{tree}(\operatorname{mcd}(\Theta))\}$. Let $\Theta$ be a nonterminal 


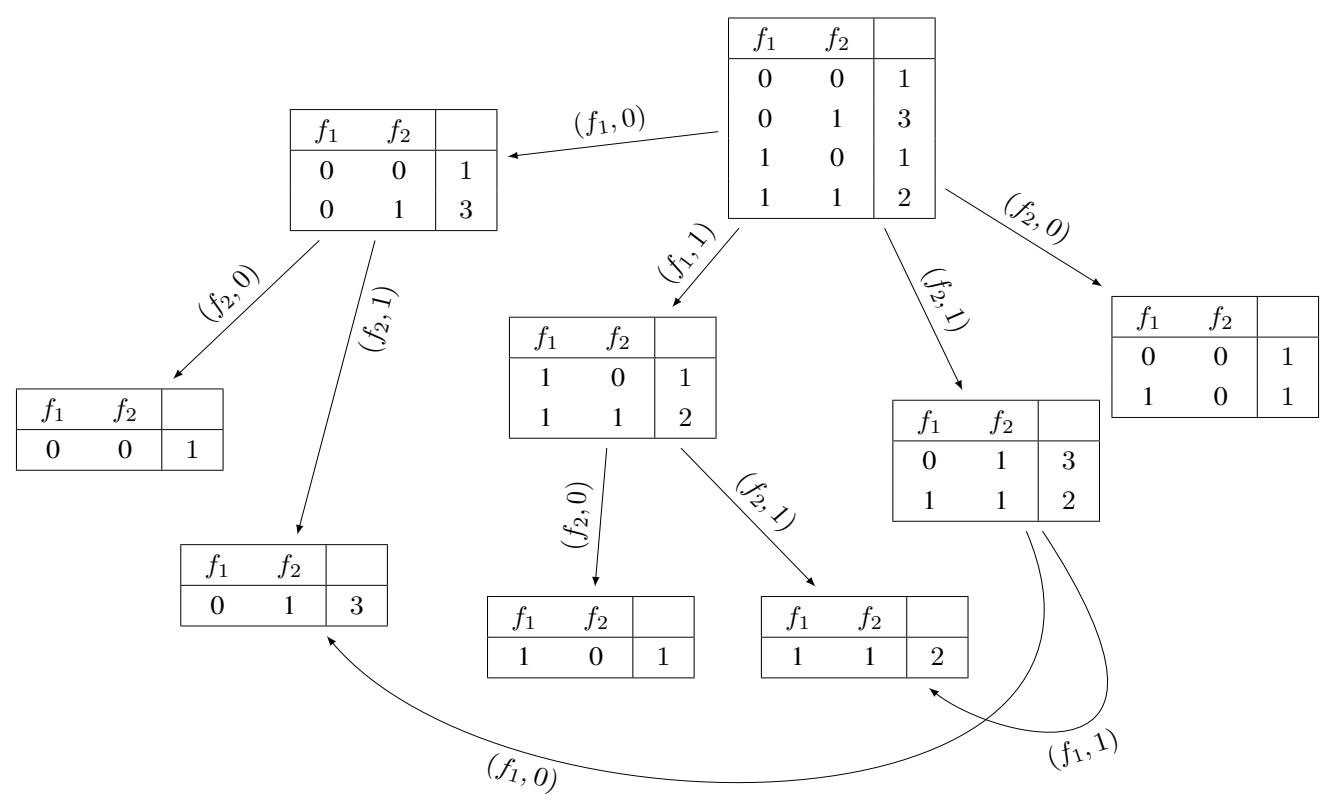

Figure 4. Directed acyclic graph $\Delta_{m e, 0}\left(T_{1}\right)$ for a decision table $T_{1}$

node of $G$ (in this case $U(\Theta)>\alpha U(T)), f_{i} \in E_{G}(\Theta)$, and $E\left(\Theta, f_{i}\right)=\left\{a_{1}, \ldots, a_{t}\right\}$. We denote $\operatorname{Tree}\left(G, \Theta, f_{i}\right)=\left\{\operatorname{tree}\left(f_{i}, a_{1}, \ldots, a_{t}, \Gamma_{1}, \ldots, \Gamma_{t}\right): \Gamma_{j} \in \operatorname{Tree}\left(G, \Theta\left(f_{i}, a_{j}\right)\right), j=1, \ldots, t\right\}$. Then

$$
\operatorname{Tree}(G, \Theta)=\bigcup_{f_{i} \in E_{G}(T)} \operatorname{Tree}\left(G, \Theta, f_{i}\right) .
$$

We will say that the graph $G$ describes the set of decision trees $\operatorname{Tree}(G, T)$.

Proposition 3.1. [13] Let $U$ be an uncertainty measure, $\alpha$ be a real number such that $0 \leq \alpha \leq 1$, and $T$ be a decision table. Then, for any node $\Theta$ of the graph $\Delta_{U, \alpha}(T)$, the following equality holds:

$$
\operatorname{Tree}\left(\Delta_{U, \alpha}(T), \Theta\right)=D T_{U, \alpha}(\Theta) .
$$

From this proposition it follows that the DAG $\Delta_{U, \alpha}(T)$ describes the set of all $(U, \alpha)$-decision trees for $T$.

\subsection{Optimization of Decision Trees}

Let $\psi$ be an increasing cost function for decision trees given by the triple of functions $\psi^{0}, F$ and $w$, $U$ be an uncertainty measure, $\alpha$ be a real number such that $0 \leq \alpha \leq 1, T$ be a decision table with $n$ conditional attributes $f_{1}, \ldots, f_{n}$, and $G$ be a bundle-preserving subgraph of the graph $\Delta_{U, \alpha}(T)$.

We consider now an algorithm $\mathcal{A}_{2}$ (a procedure of optimization relative to the cost function $\psi$ ) described in [13]. The algorithm $\mathcal{A}_{2}$ attaches to each node $\Theta$ of $G$ the number $c(\Theta)=\min \{\psi(\Theta, \Gamma)$ : 
$\Gamma \in \operatorname{Tree}(G, \Theta)\}$ and, probably, remove some $f_{i}$-bundles of edges starting from nonterminal nodes of $G$. As a result, we obtain a bundle-preserving subgraph $G^{\psi}$ of the graph $G$. It is clear that $G^{\psi}$ is also a bundle-preserving subgraph of the graph $\Delta_{\alpha}(T)$.

Algorithm $\mathcal{A}_{2}$ (procedure of decision tree optimization).

Input: A bundle-preserving subgraph $G$ of the graph $\Delta_{U, \alpha}(T)$ for some decision table $T$, uncertainty measure $U$, a real number $\alpha$ such that $0 \leq \alpha \leq 1$, and an increasing cost function $\psi$ for decision trees given by the triple of functions $\psi^{0}, F$, and $w$.

Output: The bundle-preserving subgraph $G^{\psi}$ of the graph $G$.

1. If all nodes of the graph $G$ are processed then return the obtained graph as $G^{\psi}$ and finish the work of the algorithm. Otherwise, choose a node $\Theta$ of the graph $G$ which is not processed yet and which is either a terminal node of $G$ or a nonterminal node of $G$ for which all children are processed.

2. If $\Theta$ is a terminal node then set $c(\Theta)=\psi^{0}(\Theta)$, mark node $\Theta$ as processed and proceed to step 1.

3. If $\Theta$ is a nonterminal node then, for each $f_{i} \in E_{G}(\Theta)$, compute the value

$$
c\left(\Theta, f_{i}\right)=F\left(c\left(\Theta\left(f_{i}, a_{1}\right)\right), \ldots, c\left(\Theta\left(f_{i}, a_{t}\right)\right)\right)+w(\Theta)
$$

where $\left\{a_{1}, \ldots, a_{t}\right\}=E\left(\Theta, f_{i}\right)$, and set $c(\Theta)=\min \left\{c\left(\Theta, f_{i}\right): f_{i} \in E_{G}(\Theta)\right\}$. Remove all $f_{i}$-bundles of edges starting from $\Theta$ for which $c(\Theta)<c\left(\Theta, f_{i}\right)$. Mark the node $\Theta$ as processed and proceed to step 1 .

If we apply the procedure of optimization to a bundle-preserving subgraph $G$ of the graph $\Delta_{U, \alpha}(T)$ and an increasing cost function $\psi$ then we obtain the bundle-preserving subgraph $G^{\psi}$ of the graph $G$. The bundle-preserving subgraph $G$ describes the set $A=\operatorname{Tree}(G, T)$ of decision trees for the table $T$. The bundle-preserving subgraph $G^{\psi}$ describes the set $B=\operatorname{Tree}\left(G^{\psi}, T\right)$ of decision trees for the table $T$. Let $C$ be the set of decision trees from $A$ which have the minimum value of the function $\psi$ among all trees from the set $A$. We will show that $B \subseteq C$ if $\psi$ is increasing, and $B=C$ if $\psi$ is strictly increasing.

Theorem 3.2. [13] Let $\psi$ be an increasing cost function for decision trees, $U$ be an uncertainty measure, $\alpha$ be a real number such that $0 \leq \alpha \leq 1, T$ be a decision table, and $G$ be a bundle-preserving subgraph of the graph $\Delta_{U, \alpha}(T)$. Then, $\operatorname{Tree}\left(G^{\psi}, T\right)$ is a subset of the set of decision trees from $\operatorname{Tree}(G, T)$ which have the minimum value of the function $\psi$ among all trees from the set $\operatorname{Tree}(G, T)$, and $c(T)=\min \{\psi(T, \Gamma): \Gamma \in \operatorname{Tree}(G, T)\}$.

Theorem 3.3. [13] Let $\psi$ be a strictly increasing cost function for decision trees, $U$ be an uncertainty measure, $\alpha$ be a real number such that $0 \leq \alpha \leq 1, T$ be a decision table, and $G$ be a bundlepreserving subgraph of the graph $\Delta_{U, \alpha}(T)$. Then, Tree $\left(G^{\psi}, T\right)$ is the set of decision trees from $\operatorname{Tree}(G, T)$ which have the minimum value of the function $\psi$ among all trees from the set $\operatorname{Tree}(G, T)$, and $c(T)=\min \{\psi(T, \Gamma): \Gamma \in \operatorname{Tree}(G, T)\}$. 
In [13], infinite classes of decision tables are described for which the Algorithms $\mathcal{A}_{1}$ and $\mathcal{A}_{2}$ have polynomial time complexity depending on the size of the input tables when we use uncertainty measures from the set $\{m e, r m e, e n t, g i n i, R\}$ and cost functions from the set $\{h, t p l, L\}$. In general case, the Algorithms $\mathcal{A}_{1}$ and $\mathcal{A}_{2}$ have exponential time complexity depending on the size of the input tables. However, the considered algorithms are applicable to medium-sized decision tables which allows us to use them for the experimental study of totally optimal decision trees (see Section 4).

\subsection{Multi-stage Optimization of Decision Trees and Existence of Totally Optimal De- cision Trees}

The designed tools allow us to make multi-stage optimization of decision trees relative to a sequence of cost functions since the result of application of the procedure of optimization relative to a cost function is the set of all optimal trees from the input set of decision trees if the cost function is strictly increasing, and is a subset of the set of optimal trees if a cost function is increasing. These tools also help us to recognize the existence of totally optimal trees: a totally optimal tree relative to a number of cost functions exists if and only if the results of multi-stage optimization relative to the considered cost functions do not depend on the order of cost functions.

We can make multi-stage optimization of $(U, \alpha)$-decision trees for $T$ relative to a sequence of strictly increasing cost functions $\psi_{1}, \psi_{2}, \ldots$. We begin from the graph $G=\Delta_{U, \alpha}(T)$ and apply to it the procedure of optimization relative to the cost function $\psi_{1}$ (the algorithm $\mathcal{A}_{2}$ ). As a result, we obtain a bundle-preserving subgraph $G^{\psi_{1}}$ of the graph $G$.

By Proposition 3.1, the set $\operatorname{Tree}(G, T)$ is equal to the set $D T_{U, \alpha}(T)$ of all $(U, \alpha)$-decision trees for $T$. Using Theorem 3.3, we obtain that the set $\operatorname{Tree}\left(G^{\psi_{1}}, T\right)$ coincides with the set of all decision trees from $\operatorname{Tree}(G, T)$ which have minimum cost relative to $\psi_{1}$ among all trees from the set $\operatorname{Tree}(G, T)$. Next we apply to $G^{\psi_{1}}$ the procedure of optimization relative to the cost function $\psi_{2}$. As a result, we obtain a bundle-preserving subgraph $G^{\psi_{1}, \psi_{2}}$ of the graph $G^{\psi_{1}}$ (and of the graph $G=\Delta_{\alpha}(T)$ ). By Theorem 3.3, the set $\operatorname{Tree}\left(G^{\psi_{1}, \psi_{2}}, T\right)$ coincides with the set of all decision trees from $\operatorname{Tree}\left(G^{\psi_{1}}, T\right)$ which have minimum cost relative to $\psi_{2}$ among all trees from $\operatorname{Tree}\left(G^{\psi_{1}}, T\right)$, etc.

If one of the cost functions $\psi_{i}$ is increasing and not strictly increasing then the $\operatorname{set} \operatorname{Tree}\left(G^{\psi_{1}, \ldots, \psi_{i}}, T\right)$ is a subset of the set of all decision trees from $\operatorname{Tree}\left(G^{\psi_{1}, \ldots, \psi_{i-1}}, T\right)$ that have minimum cost relative to $\psi_{i}$ among all trees from $\operatorname{Tree}\left(G^{\psi_{1}, \ldots, \psi_{i-1}}, T\right)$.

Assume that $\psi_{1}, \ldots, \psi_{m-1}$ are strictly increasing cost functions and $\psi_{m}$ is increasing or strictly increasing. We now describe how to recognize the existence of a $(U, \alpha)$-decision tree for $T$ which is a totally optimal $(U, \alpha)$-decision tree for $T$ relative to the cost functions $\psi_{1}, \ldots, \psi_{m}$.

First, we construct the graph $G=\Delta_{U, \alpha}(T)$ using the algorithm $\mathcal{A}_{1}$. For $i=1, \ldots, m$, we apply to $G$ the procedure of optimization relative to $\psi_{i}$ (the Algorithm $\mathcal{A}_{2}$ ). As a result, we obtain for $i=1, \ldots, m$, the graph $G^{\psi_{i}}$ and the number $\psi_{i}^{U, \alpha}(T)$ attached to the node $T$ of $G^{\psi_{i}}$. Next, we apply to $G$ sequentially the procedures of optimization relative to the cost functions $\psi_{1}, \ldots, \psi_{m}$. As a result, we obtain graphs $G^{\psi_{1}}, G^{\psi_{1}, \psi_{2}}, \ldots, G^{\psi_{1}, \ldots, \psi_{m}}$ and numbers $\varphi_{1}, \varphi_{2}, \ldots, \varphi_{m}$ attached to the node $T$ of these graphs. It is clear that $\varphi_{1}=\psi_{1}^{U, \alpha}(T)$. For $i=2, \ldots, m, \varphi_{i}=\min \left\{\psi_{i}(T, \Gamma): \Gamma \in\right.$ $\left.\operatorname{Tree}\left(G^{\psi_{1}, \ldots, \psi_{i-1}}, T\right)\right\}$. One can show that a totally optimal $(U, \alpha)$-decision tree for $T$ relative to the cost functions $\psi_{1}, \ldots, \psi_{m}$ exists if and only if $\varphi_{i}=\psi_{i}^{U, \alpha}(T)$ for $i=1, \ldots, m$. 


\section{Experimental Results}

In this section, we consider results of computer experiments with nine decision tables from [7]. Before the experimental work, some preprocessing procedures are performed. A conditional attribute is removed if it has unique value for each row. The missing value for an attribute is filled up with the most common value for this attribute. In some tables, there are equal rows with, possibly, different decisions. In this case, each group of equal rows is replaced with a single row from the group with the most common decision for this group. As a result, we obtain consistent decision tables without missing values (a decision table is called consistent if it has no equal rows with different decisions). The nine decision tables used in experiments are described in Table 1. The first column 'Decision table' refers to the name of the decision table from [7], the second column 'Rows' refers to the number of rows, and the last column 'Attributes' refers to the number of attributes (conditional and decision).

Table 1. Decision tables used in experiments

\begin{tabular}{|c|c|c|}
\hline Decision table & Rows & Attributes \\
\hline BALANCE-SCALE & 625 & 5 \\
\hline BREAST-CANCER & 266 & 10 \\
\hline CARS & 1728 & 7 \\
\hline HAYES-ROTH-DATA & 69 & 5 \\
\hline HOUSE-VOTES-84 & 279 & 17 \\
\hline LYMPHOGRAPHY & 148 & 19 \\
\hline SOYBEAN-SMALL & 47 & 36 \\
\hline TIC-TAC-TOE & 958 & 10 \\
\hline ZOO-DATA & 59 & 17 \\
\hline
\end{tabular}

For each decision table $T$, each uncertainty measure $U \in\{$ ent, gini, me, rme, $R\}$, each $\alpha \in$ $\{0.00,0.01,0.02, \ldots, 1.00\}$, and each pair of different cost functions $\psi_{1}, \psi_{2} \in\left\{h, h_{\text {avg }}, L\right\}$, we check if there exists a totally optimal $(U, \alpha)$-decision tree for $T$ relative to the cost functions $\psi_{1}, \psi_{2}$. We check also if there exists a totally optimal $(U, \alpha)$-decision tree for $T$ relative to the cost functions $h, h_{\text {avg }}, L$.

The results of experiments can be found in Figs. 5, 6, 7, 8, and 9 for uncertainty measures ent, gini, me, rme, and $R$, respectively.

In each figure,

- The graph 'havg_h' shows, for the uncertainty measure $U$ considered in this figure and each $\alpha \in\{0.00,0.01,0.02, \ldots, 1.00\}$, the number of decision tables $T$ for each of which there exists a totally optimal $(U, \alpha)$-decision tree for $T$ relative to the cost functions $h_{\text {avg }}, h$.

- The graph 'havg_L' shows, for the uncertainty measure $U$ considered in this figure and each $\alpha \in\{0.00,0.01,0.02, \ldots, 1.00\}$, the number of decision tables $T$ for each of which there exists a totally optimal $(U, \alpha)$-decision tree for $T$ relative to the cost functions $h_{a v g}, L$. 
- The graph 'L_h' shows, for the uncertainty measure $U$ considered in this figure and each $\alpha \in$ $\{0.00,0.01,0.02, \ldots, 1.00\}$, the number of decision tables $T$ for each of which there exists a totally optimal $(U, \alpha)$-decision tree for $T$ relative to the cost functions $L, h$.

- The graph 'h_L_havg' shows, for the uncertainty measure $U$ considered in this figure and each $\alpha \in\{0.00,0.01,0.02, \ldots, 1.00\}$, the number of decision tables $T$ for each of which there exists a totally optimal $(U, \alpha)$-decision tree for $T$ relative to the cost functions $h, L, h_{\text {avg }}$.

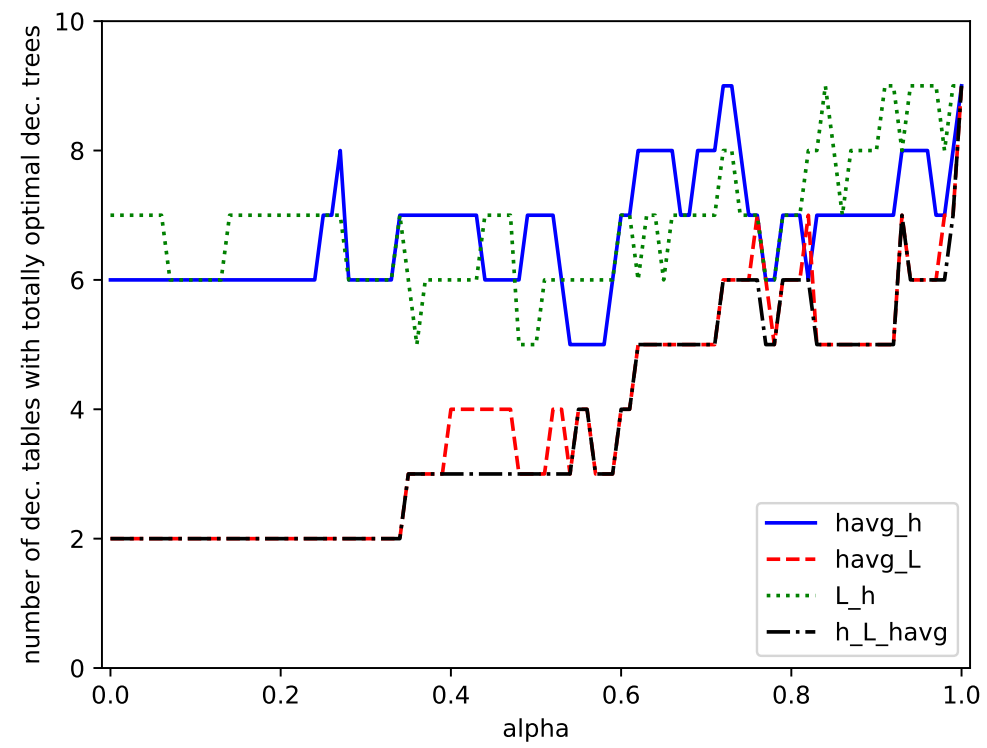

Figure 5. Number of decision tables with totally optimal decision trees for uncertainty measure ent

The obtained experimental results show that totally optimal decision trees exist in many cases. There are some additional observations:

- In Figs. 5-9, the behavior of lines is mainly irregular. The only exception is the line 'havg_h' for the uncertainty measure $R$.

- Some lines have upward trend: lines 'havg_h' and 'L_h' for uncertainty measures me and $R$; lines 'havg_L' and ' $h \_L \_h a v g$ ' for each of the considered uncertainty measures.

- In almost all cases, the lines 'havg_L' are below the lines 'havg_h' and 'L_h'or coincide with these lines. This result is consistent with the results obtained in [10] for totally optimal decision trees computing Boolean functions.

- In all cases, the lines 'h_L_havg' are below the lines 'havg_L' or coincide with these lines. This result is predictable. 


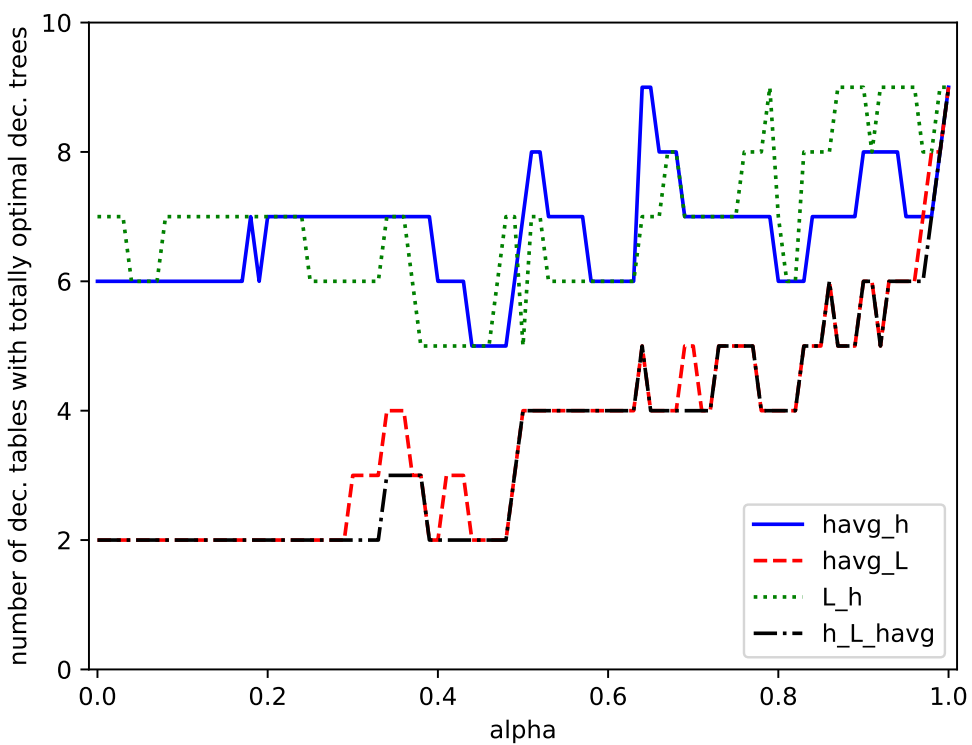

Figure 6. Number of decision tables with totally optimal decision trees for uncertainty measure gini

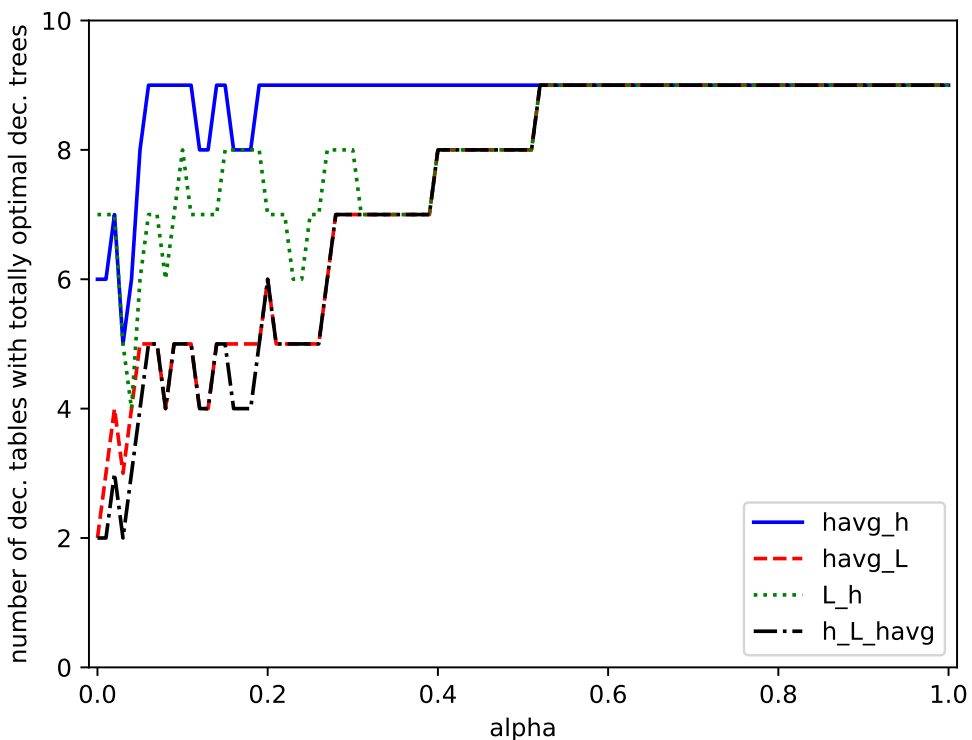

Figure 7. Number of decision tables with totally optimal decision trees for uncertainty measure me 


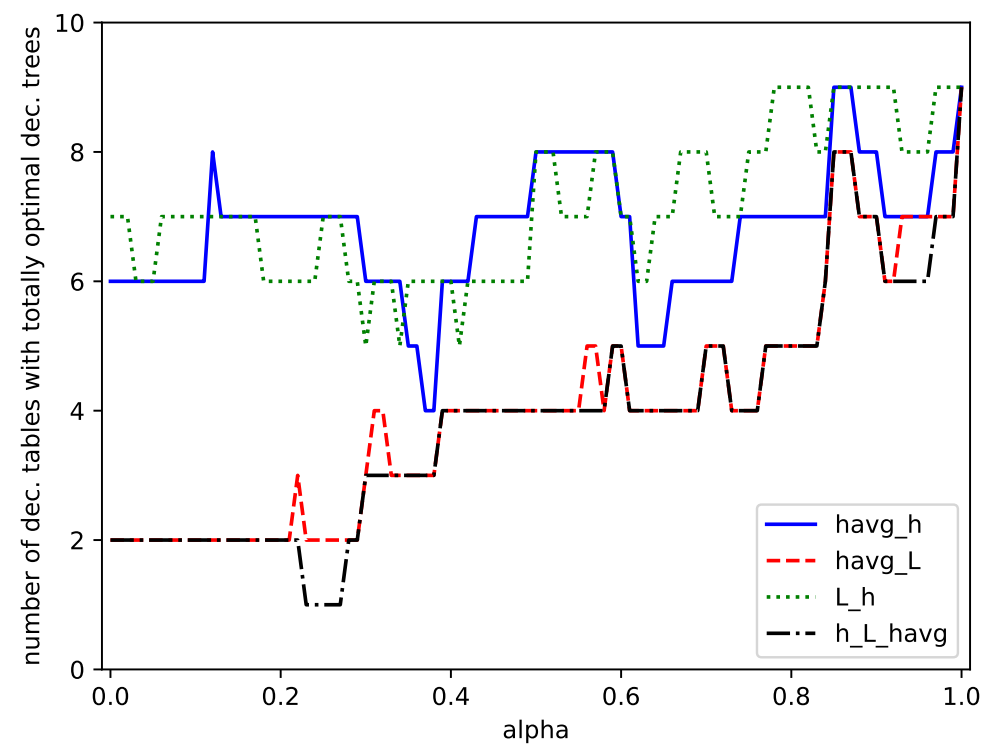

Figure 8. Number of decision tables with totally optimal decision trees for uncertainty measure $r m e$

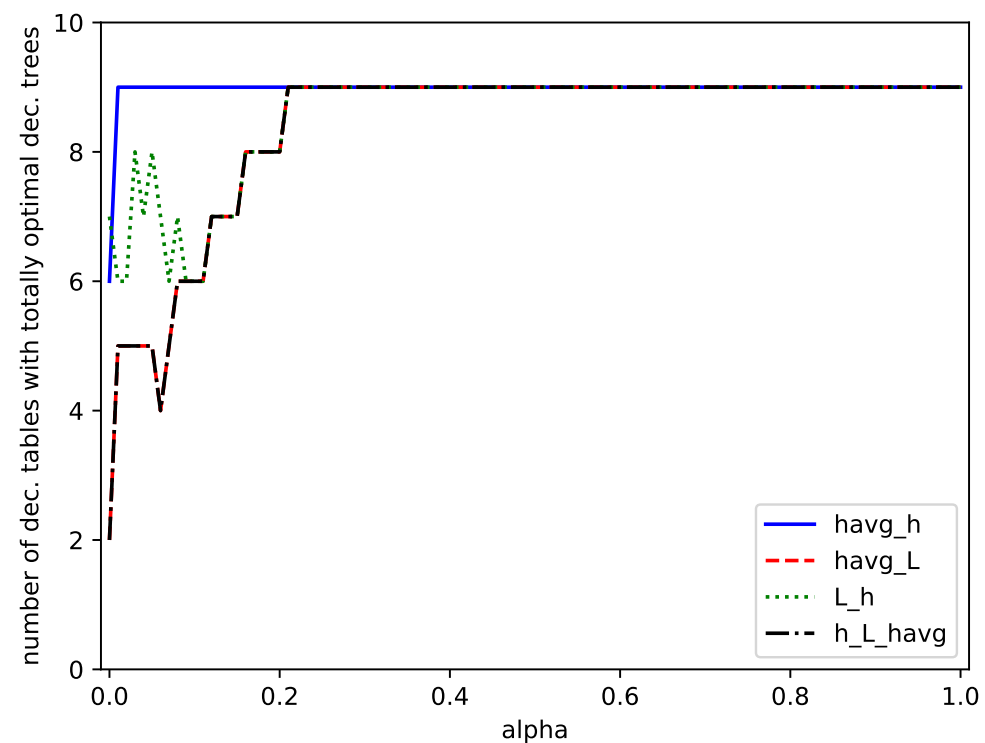

Figure 9. Number of decision tables with totally optimal decision trees for uncertainty measure $R$ 


\section{Conclusions}

In this paper, we experimentally studied the existence of totally optimal $(U, \alpha)$-decision trees for five uncertainty measures $U$ and nine decision tables from the UCI Machine Learning Repository [7]. Totally optimal $(U, \alpha)$-decision trees exist in many cases. Lines, describing how the number of decision tables with totally optimal $(U, \alpha)$-decision trees grows with the increasing of $\alpha$, have often upward trend but the behavior of these lines is mainly irregular. In the future, we are planning to continue the study of totally optimal trees since they can be useful in various applications related to problem solving and knowledge representation.

\section{Acknowledgments}

Research reported in this publication was supported by King Abdullah University of Science and Technology (KAUST). We are greatly indebted to the anonymous reviewers for useful comments and suggestions.

\section{References}

[1] Moshkov M. Time complexity of decision trees. In: Peters JF, Skowron A (eds.), Trans. Rough Sets III, volume 3400 of Lecture Notes in Computer Science, pp. 244-459. Springer, 2005.

[2] Rokach L, Maimon O. Data Mining with Decision Trees: Theory and Applications. World Scientific Publishing, River Edge, NJ, 2008.

[3] Azad M, Chikalov I, Hussain S, Moshkov M. Multi-pruning of decision trees for knowledge representation and classification. In: 3rd IAPR Asian Conference on Pattern Recognition, ACPR 2015, Kuala Lumpur, Malaysia, November 3-6, 2015. IEEE, 2015 pp. 604-608.

[4] Moshkov M, Chikalov I. Consecutive optimization of decision trees concerning various complexity measures. Fundam. Inform., 2004. 61(2):87-96.

[5] Alkhalid A, Amin T, Chikalov I, Hussain S, Moshkov M, Zielosko B. DAGGER: a tool for analysis and optimization of decision trees and rules. In: Ficarra FVC, Kratky A, Veltman KH, Ficarra MC, Nicol E, Brie M (eds.), Computational Informatics, Social Factors and New Information Technologies: Hypermedia Perspectives and Avant-Garde Experiencies in the Era of Communicability Expansion, pp. 29-39. Blue Herons, 2011.

[6] Alkhalid A, Amin T, Chikalov I, Hussain S, Moshkov M, Zielosko B. Optimization and analysis of decision trees and rules: dynamic programming approach. International Journal of General Systems, 2013. 42(6):614-634.

[7] Lichman M. UCI Machine Learning Repository. University of California, Irvine, School of Information and Computer Sciences, 2013. URL http://archive.ics.uci.edu/ml.

[8] Pawlak Z. Rough Sets - Theoretical Aspect of Reasoning About Data. Kluwer Academic Publishers, Dordrecht, 1991.

[9] Pawlak Z, Skowron A. Rudiments of rough sets. Information Sciences, 2007. 177(1):3-27.

[10] Chikalov I, Hussain S, Moshkov M. Totally optimal decision trees for Boolean functions. Discrete Applied Mathematics, 2016. 215:1-13. 
[11] Azad M, Moshkov M. Multi-stage optimization of decision and inhibitory trees for decision tables with many-valued decisions. European Journal of Operational Research, 2017. 263(3):910-921.

[12] Aldilaijan A, Azad M, Moshkov M. Experimental study of totally optimal decision trees. In: 26th International Workshop on Concurrency, Specification and Programming, Warsaw, Poland, September 25-27, 2017 (to appear) .

[13] AbouEisha H, Amin T, Chikalov I, Hussain S, Moshkov M. Extensions of Dynamic Programming for Combinatorial Optimization and Data Mining, volume 146 of Intelligent Systems Reference Library. Springer, 2019. 